\title{
COVID-19 and Diabetes Among Comorbidities: A Systematic Review
}

\section{Gurbuz P*}

Inonu University, Vocational School of Health Services, Malatya, Turkey

*Correspondence: Perihan Gurbuz, Inonu University, Vocational School of Health Services, Malatya, Turkey

Received on 31 May 2020; Accepted on 23 July 2020; Published on 30 July 2020

Copyright $(02020$ Gurbuz P. This is an open access article and is distributed under the Creative Commons Attribution License, which permits unrestricted use, distribution, and reproduction in any medium, provided the original work is properly cited.

\begin{abstract}
The world has been facing a novel coronavirus, COVID-19 pandemic since the beginning of 2020. Until the end of May, 5.9 million confirmed cases and 350.000 deaths have been reported. Diabetes, as a prevalent chronic disease is known to be a risk factor for infection onset and disease severity. In this study, a systematic review has been planned to determine the relation between COVID-19 and diabetes among other comorbidities. For this aim, 564 researches have been determined about the topic and 48 of them have been evaluated in the review. The researches have been done with 91.172 COVID-19 patients, and diabetes ratio among the researches differ from 3.3\% to 40\%. Besides, age, hypertension, cardiovascular diseases, smoking status, and respiratory diseases have been evaluated in the review as common comorbidities. As a result of the study, diabetes and hypertension have been determined to be important risk factors in COVID-19 onset and severity. However, further detailed multidisciplinary researches about COVID-19, diabetes and comorbidities will be valuable in the COVID-19 pandemic process and future aspects.
\end{abstract}

Keywords: COVID-19, diabetes, systematic evaluation

Abbreviations: WHO: World Health Organization; HT: hypertension; CVD: cardiovascular disease

\section{Background}

A novel coronavirus (SARS-CoV-2) infection as named COVID-19, which firstly begun in Wuhan, China has entered the world literature by December 2019. Besides being asymptomatic in general, severe pneumonia and fatality ratios were also realized to be very high, and the infection spread all over the world in a small period of time [1]. World Health Organization (WHO) declared the infection as COVID-19 pandemic on March 11, 2020. By the day (26.05.2020); global death number is over 350.000 and there are nearly 5.9 million confirmed cases [2]. 
Diabetes, as a widespread chronic disease which affects the whole organism, is considered as an epidemic of the 21 st century by patient number increase of 108 million to 422 million from 1980 to 2014 [3]. Also, this number is predicted to be 642 million by the year 2040 [4]. As a chronic disease, diabetes causes various acute and chronic complications [5]. Besides, it has been shown to increase infection and infectious complication susceptibility in patients [6-8]. Comorbidities as; diabetes, chronic heart diseases, chronic pulmonary diseases and smoking are considered as important risk factors in acute and chronic outcomes of respiratory system diseases including pneumonia [9]. Among the other comorbidities, diabetes had been detected to be a negative factor in disease process of the two recent coronavirus epidemics, SARS, and MERS [7, 10]. Likewise, mentioned comorbidities have been shown to be important risk factors for complications and fatality in COVID-19 pandemic [10, 11].

By this point, evaluation of COVID-19 and diabetes relation will be important in COVID-19 road map. For this purpose, a systematic review to determine, whether there exists a relation between COVID-19 and diabetes among other comorbidities, has been planned in this study.

\section{Methods}

This systematic review has been done to analyze the current literature about COVID-19 and diabetes relation. For the review 'The Preferred Reporting Items for Systematic Reviews and Meta-Analysis' (PRISMA) guidelines were used.

Eligibility criteria for research selection were determined to be:

- $\quad$ Clinical trials and researches about COVID-19 and diabetes.

- $\quad$ Published articles, pre-print researches, and reports of currently undergoing researches.

- $\quad$ No age and geographical location distinction.

The primary outcome of the study was to determine COVID-19 and diabetes relation. Electronic search was done on PubMed and Springer Link databases, with the keywords of COVID-19, epidemiological, clinical characteristics, comorbidities, and diabetes (Figure 1).

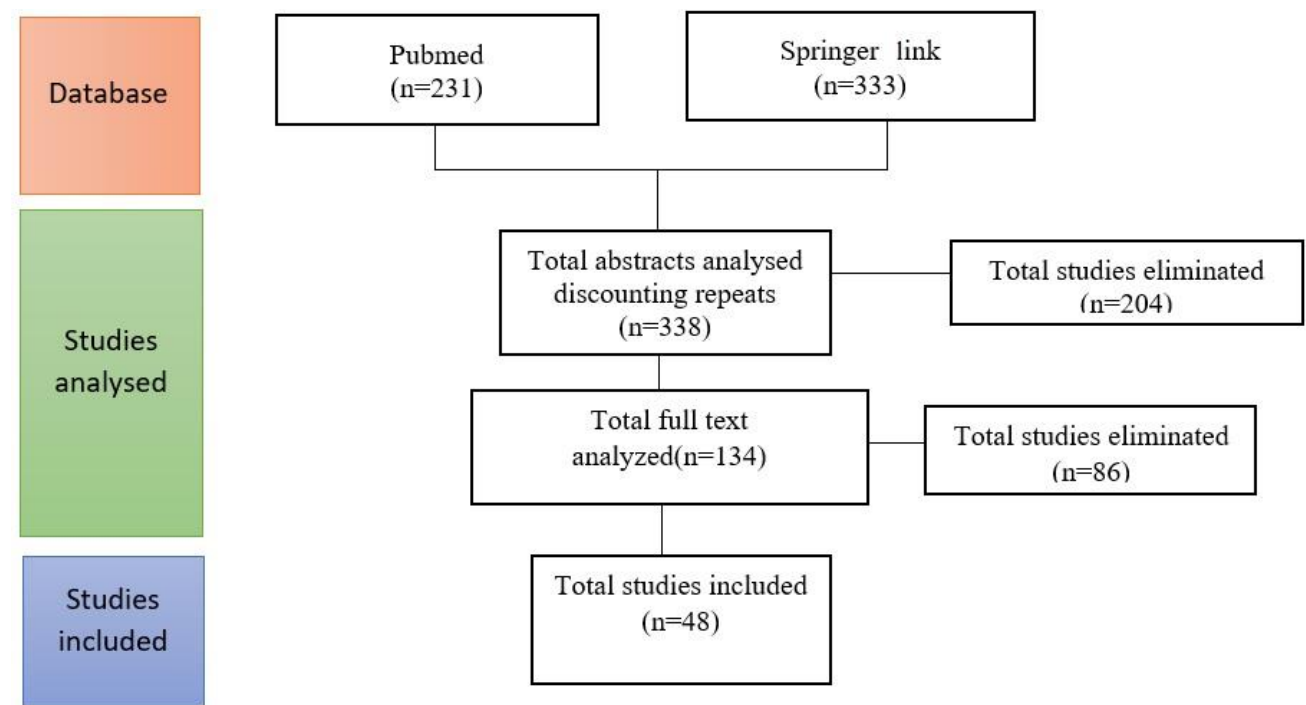

Figure 1: Flowchart detailing study selection.

The results of the databases were evaluated by the author on May 10-20, 2020. In order to minimalize the error and bias risks, this procedure was performed two times with ten days interval. To obtain the researches about the topic; the titles and abstracts were screened, duplications were detected and removed, the manuscripts of the researches 
which met eligibility criteria were evaluated for the detailed data, and the appropriate researches were included to the study (Figure 1).

\section{Results}

In the two-independent scannings (May 10 and May 20, 2020), 564 researches have been obtained from PubMed and Springer Link databases. Of the obtained researches, 231 were duplicate and 204 did not meet the eligibility criteria. 134 researches were further investigated by full text analyzing. At the end, 48 researches meeting the criteria were included in the study. The findings of the included researches were categorized according to; prevalence of diabetes, hypertension, cardiovascular disease, smoking status and respiratory diseases in COVID-19 (Table 1), prevalence of diabetes in non-severe and severe COVID-19 patients (Table 2), prevalence of diabetes in survivor and non-survivor COVID-19 patients (Table 3).

\section{Discussion and Conclusion}

Diabetes increases infection risk by the factors as; neutrophil dysfunction, decreased T-cell response, and poor humoral immunity [8]. Findings about diabetes and other comorbidities' prevalence and mechanism of action in the novel COVID-19 pandemic will be important in shaping future strategies.

When the 48 included researches in this systematic review are evaluated, it is seen that; 35 have been made in China, 5 have been made in USA, 2 have been made in Italy, and there exists one study from Spain, France, Iran, Israel, South Korea and Belgium. The researches totally include 91.172 COVID-19 patients.

Diabetes prevalence in the researches differ from $3.3 \%$ to $40 \%[19,44]$. When the researches including only fatal cases are considered, this ratio is between $22.4 \%$ to $40 \%[44,55]$. WHO reports diabetes prevalence as $8.5 \%$ in adults [3]. Although the diabetic patient ratio differs in a wide range, it is under than the predicted ratio in only 11 researches, while this ratio is over $20 \%$ in 15 included researches of the review. As Gentile et al.'s findings of nearly doubled ratios of $>80$ years of age diabetic patient percentages, many of the researches indicate statistically significant increased diabetes prevalence among COVID-19 patients (Table 1) [30]. Furthermore, Xiong et al. and Chow et al. have reported diabetes ratio to be higher in symptomatic COVID-19 patients, and this is an important point to focus on about diabetes and COVID-19 relation [35, 38]. Differences among the researches may be due to the included patient heterogeneity but achieved results of the review simply predicts diabetes as a potential risk factor for COVID19 infection.

When COVID-19 infection correlation with diabetes is evaluated, it is seen that diabetes has been detected to be a risk factor in 17 of the included researches [6, 7, 13-16, 28, 31, 36, 37, 41, 45, 47, 49, 52-54]. Among them; Huang et al. have reported diabetes to be an independent risk factor and Garg et al. have shown diabetes to be a risk factor for COVID-19 severity in all ages $[45,53]$. As an important point to focus on, Huang et al., Ren et al. and Deng et al. have reported diabetes to be the only risk factor for COVID-19 among other comorbidities [18, 20, 21].

In the review, diabetic COVID-19 patients' illness severity has been compared in 18 researches (Table 2). When the mentioned researches are evaluated, it is seen that COVID-19 infection severity is statistically higher in diabetics in 8 of the researches [13, 15, 20, 27, 36, 37, 47, 53], while 4 researches did not find a significance [34, 35, 41, 46]. According to Guo et al.'s research, diabetic COVID-19 patients without other comorbidities not only had severe pneumonia risk, but also had; increased release of injury-related enzymes, uncontrolled severe inflammatory responses, glucose metabolism dysregulation associated hypercoagulable state, and increased levels of inflammationrelated biomarkers (C-reactive protein, IL-6, D-dimer, serum ferritin and coagulation index) [6]. Likewise, in their research with 7337 patients including 952 pre-existing type 2 diabetics, Zhu et al. have determined that, while diabetic patients needed more medical interventions and had multiple organ injury, well-controlled blood glucose levels (3.9 to $10.0 \mathrm{mmol} / \mathrm{L}$ ) indicated lower mortality [7]. 
There exist several factors in determining diabetes and infection relation, as Sirtuin 1 repression and IL-17 expression $[10,58-60]$. When the researches in this review are evaluated; blood glucose level regulation has been shown to be a very important determinant in many of the studies [7, 48], also Ren et al. have determined TyG index (proposed to be an insulin resistance marker) to be associated with COVID-19 severity and mortality [13].

Also, when looked from the inverse side, infections are important risk factors for acute hyperglycemia and diabetic complications [61], and there exists several reports about ketosis, hyperosmolar hyperglycemic state, and COVID-19 severity [62-64]. Likewise, among the included researches, Bode et al. have reported the hyperglycemia ratio in COVID-19 patients without a pre-diagnosis of diabetes to be nearly $20 \%$ (with increased morbidity and mortality prevalence), besides they have determined acute hypoglycemia ratios to be higher in diabetic and/or uncontrolled hyperglycemic patients [48].

There exist 9 researches about the relation of diabetes with COVID-19 mortality ratios (Table 3). Among them, in 4 of the researches diabetes has been found to increase fatality [16, 17, 23, 43], while 2 researches determined no relation $[29,51]$. Besides, 6 researches, which is not included to 'Table 3', have stated diabetes to be related to mortality for COVID-19 infection [7, 18, 39, 42, 44, 48]. Among them, Bode et al. determined mortality ratio to be four-fold higher in diabetic and/or uncontrolled hyperglycemic patients [48].

Hypertension (HT) is known to be a risk factor for disease onset and severity of infection diseases [65, 66]. In this review HT ratios varies between $1.99 \%$ to $76 \%$ [33, 57]. However, the smallest ratio of mortality-based researches is $37.6 \%$ [55]. While 10 researches in the review have reported HT to be a risk factor for COVID-19 onset [13, 16, 17, 21, 23, 24, 27, 28, 43, 44], Wu et al. and Leung $\mathrm{C}$ did not find any relation [36, 39]. These findings indicate HT an important risk factor for COVID-19 infection.

Cardiovascular disease (CVD) ratios have been determined to be $1.3 \%$ to $68.7 \%$ in the included researches [35, 49]. When the researches about mortality in COVID-19 are evaluated, the smallest ratio is 11.8\% [55]. CVD has been determined to be related with COVID-19 onset and severity in 8 researches $[17,21,27,29,31,35,47,51]$. Among them; Wang et al., Deng et al. and Ruan et al. have determined CVD disease to increase COVID-19 mortality rates and Xiong et al. have determined CVD to be the only comorbidity effecting COVID-19 onset [17, 21, 29, 35].

In the evaluation of gender and age, 5 researches reported male gender [13, 16, 21, 27, 47], and 7 researches determined age as risk factors for COVID-19 infection [13, 15-17, 24, 44, 47]. While smoking rates were determined between $1.3 \%$ and $32.5 \%$, no relation was obtained between smoking and COVID-19 disease in the included researches [38, 57]. Also, there was only one research that reported pulmonary diseases as a risk factor for COVID-19 infection [51].

When the mentioned comorbidities are evaluated as a whole, they have not only been reported to increase the onset and severity of COVID-19 infection but also have been determined to cause poorer outcomes [50, 52, 56, 57]. Also, diabetes and HT reveal as the most common comorbidities that effect the COVID-19 morbidity and mortality rates when compared with the other comorbidities. These findings show noteworthy similarities with the former studies about comorbidities, diabetes, infection, and COVID-19 [1, 8, 66-71]. However, there exists important limitations as, lack of homogeneity of patient groups and increased ratios of undiagnosed diabetes patients. By this point, specified researches about; the diabetes' role among other comorbidities, diabetic complications, hyperglycemia ratios, insulin need and usage modalities, and new-onset diabetes in COVID-19 patients will be valuable to understand the topic in a more detailed manner. 


\begin{tabular}{|c|c|c|c|c|c|c|c|c|c|}
\hline Author/Country & $\begin{array}{l}\text { Date(day, month, } \\
\text { year) }\end{array}$ & $\mathbf{n}$ & Age & $\begin{array}{l}\mathrm{DM} \\
\mathrm{n}(\%) \\
\end{array}$ & $\begin{array}{l}\text { HT } \\
\text { n }(\%) \\
\end{array}$ & $\begin{array}{l}\text { CVD } \\
\text { n }(\%) \\
\end{array}$ & $\begin{array}{l}\text { Smoker } \\
\text { n }(\%)\end{array}$ & $\begin{array}{l}\text { RD } \\
\text { n }(\%)\end{array}$ & Ref \\
\hline Lian J et al./China & $17.01 .20-07.02 .20$ & 788 & 45.8 & $57(7.23)$ & $126(15.98)$ & $11(1.39)$ & $54(6.85)$ & $9(1,14)$ & [12] \\
\hline Ren $\mathrm{H}$ et al./China & $12.01 .20-13.02 .20$ & 151 & 59.5 & $39(25.8)$ & $60(39.7)$ & $16(10.6)$ & NR & $2(1.3)$ & [13] \\
\hline Yuan J et al./China & 11.01.20-04.02.20 & 94 & 40 & $5(5.30)$ & $9(9.60)$ & $6(6.40)$ & NR & NR & {$[14]$} \\
\hline Chen Q et al./China & $01.01 .20-11.03 .20$ & 145 & 47.5 & $14(9.65)$ & $22(15.17)$ & NR & $6(4.13)$ & $15(10.34)$ & [15] \\
\hline Tu W et al./China & $03.01 .20-24.02 .20$ & 174 & 53.7 & $17(9.77)$ & $37(21.26)$ & NR & NR & $12(6.89)$ & [16] \\
\hline Wang D et al./China & NR-10.02.20 & 107 & 51 & $11(10.3)$ & $26(24.3)$ & $13(12.1)$ & NR & $3(2.8)$ & [17] \\
\hline Huang Q et al./China & $17.01 .20-10.02 .20$ & 54 & 41 & $5(9.3)$ & $8(14.8)$ & $8(14.8)$ & NR & $2(3.7)$ & [18] \\
\hline Zhao XY et al./China & $16.01 .20-10.02 .20$ & 91 & 46 & $3(3.3)$ & NR & NR & NR & $2(2.2)$ & [19] \\
\hline Ren D et al./China & 11.01.20-12.02.20 & 150 & 54 & $12(8)$ & $25(16.7)$ & $8(5.3)$ & $3(2)$ & $4(2.7)$ & [20] \\
\hline Deng $\mathrm{G}$ et al./China & NR-11.02.20 & 44,672 & NR & $1102(5.3)$ & $2683(12.8)$ & $873(4.2)$ & NR & $511(2.4)$ & {$[21]$} \\
\hline Liu J et al./China & NR-16.02.20 & 24 & 57.9 & $2(8)$ & $7(29)$ & $3(12.5)$ & $2(8)$ & NR & {$[22]^{*}$} \\
\hline Zhao X et al./China & $07.01 .20-28.02 .20$ & 532 & 49.5 & $59(11.09)$ & $108(20.30)$ & NR & NR & NR & {$[23]^{€}$} \\
\hline Helms J et al./France & $03.03 .20-31.03 .20$ & 150 & 63 & $30(20)$ & NR & NR & NR & $21(14)$ & {$[24]^{\beta}$} \\
\hline Pavoni V et al./Italy & $28.02 .20-10.04 .20$ & 40 & 61 & $16(40)$ & $16(40)$ & $12(30)$ & NR & $4(10)$ & {$[25]$} \\
\hline Zhang L et al./China & $17.02 .20-19.03 .20$ & 20 & 71.2 & $3(15)$ & $10(50)$ & $4(20)$ & NR & $1(5)$ & {$[26]^{\#}$} \\
\hline Shi Y et al./China & NR-17.02.20 & 487 & 46 & $29(6.0)$ & $99(20.3)$ & $11(2.3)$ & $40(8.2)$ & NR & {$[27]$} \\
\hline Zhang J et al./China & $16.01 .20-20.02 .20$ & 19 & 73 & $4(21.05)$ & $11(57.9)$ & $3(15.8)$ & NR & $3(15.8)$ & {$[28]$} \\
\hline Ruan Q et al./China & NR & 150 & 57.7 & $25(16.7)$ & $52(34.7)$ & $13(8.7)$ & NR & $3(2)$ & {$[29]^{€}$} \\
\hline Gentile $\mathrm{S}$ et al./Italy & NR & 1102 & NR & $347(31.5)$ & $794(72.1)$ & $302(27.4)$ & NR & $202(18.3)$ & {$[30] \therefore$} \\
\hline Lian $\mathrm{J}$ et al./China & $17.01 .20-31.01 .20$ & 465 & 45 & $28(6.02)$ & $82(17.63)$ & $3(0.65)$ & $60(12.90)$ & 0 & [31] \\
\hline Wan S et al./China & $23.01 .20-08.02 .20$ & 135 & 47 & $12(8.9)$ & $13(9.6)$ & $7(5.2)$ & $9(6.7)$ & $1(0.7)$ & [32] \\
\hline Nikpouraghdam M et al./Iran & $19.02 .20-15.04 .20$ & 2964 & 55.5 & $113(3.81)$ & $59(1.99)$ & $37(1.25)$ & NR & $60(2.02)$ & [33] \\
\hline Huang $\mathrm{C}$ et al./China & $16.12 .19-02.01 .20$ & 41 & 49 & $8(20)$ & $6(15)$ & $6(15)$ & $3(7)$ & $1(2)$ & {$[34]$} \\
\hline Xiong F et al./China & $01.01 .20-10.03 .20$ & 131 & 63.3 & $30(22.9)$ & NR & $90(68.7)$ & $39(29.8)$ & $5(3.8)$ & {$[35]^{\alpha}$} \\
\hline Wu C et al./China & $25.12 .19-26.01 .20$ & 201 & 51 & $22(10.9)$ & $39(19.4)$ & $8(4.0)$ & NR & $5(2.5)$ & {$[36]$} \\
\hline Itelman E et al./Israel & $20.02 .20-10.04 .20$ & 162 & 52 & $30(18.5)$ & $49(30.2)$ & $12(7.4)$ & $11(8.9)$ & $2(1.2)$ & [37] \\
\hline Chow $\mathrm{N}$ et al./USA & $12.02 .20-28.03 .20$ & 7162 & NR & $784(10.9)$ & $113(1.57)$ & $647(9.0)$ & $96(1.3)$ & $656(9.2)$ & {$[38]^{: €}$} \\
\hline Leung C/China & 26.11.19-29.01.20 & 46 & 70.6 & $11(26.2)$ & $17(40.5)$ & NR & NR & $7(16.3)$ & {$[39]^{:}$} \\
\hline De Abajo et al./Spain & $01.03 .20-24.03 .20$ & 1139 & 69.1 & $310(27.2)$ & $617(54.2)$ & $119(10.5)$ & NR & $119(10.5)$ & {$[40]$} \\
\hline Hong KS et al./South Korea & NR-29.03.20 & 98 & 55.4 & $9(9.2)$ & $30(30.6)$ & $11(11.2)$ & NR & $3(3.1)$ & [41] \\
\hline Richardson $\mathrm{S}$ et al./USA & 01.03.20-04.04.20 & 5700 & 63 & $1808(33.8)$ & $3026(56.6)$ & $595(11.1)$ & NR & $766(13.4)$ & {$[42]^{€}$} \\
\hline Zhou F et al./China & 29.12.19-31.01.20 & 191 & 56 & $36(19)$ & $58(30)$ & $15(8)$ & $11(6)$ & $6(3)$ & [43] \\
\hline Li X et al./China & 14.01.20-13.02.20 & 25 & NR & $10(40)$ & $16(64)$ & $8(32)$ & NR & $2(8)$ & {$[44] \therefore$} \\
\hline Garg $\mathrm{S}$ et al./USA & $01.03 .20-30.03 .20$ & 178 & NR & $47(28.3)$ & $79(49.7)$ & $45(27.8)$ & NR & $55(34.6)$ & [45] \\
\hline
\end{tabular}




\begin{tabular}{|c|c|c|c|c|c|c|c|c|c|}
\hline Zhang J et al./China & 16.01.20-03.02.20 & 140 & 57 & $17(12.1)$ & $42(30.0)$ & $7(5.0)$ & $2(1.4)$ & $2(1.4)$ & [46] \\
\hline Wang $\mathrm{X}$ et al/China & $07.02 .20-12.02 .20$ & 1012 & 50 & $27(2.7)$ & $46(4.5)$ & $15(1.5)$ & NR & $20(2.0)$ & [47] \\
\hline Bode B et al./USA & $01.03 .20-06.04 .20$ & 1122 & NR & $194(17.3)$ & NR & NR & NR & NR & [48] \\
\hline Zheng Y et al./China & $16.01 .20-04.02 .20$ & 73 & 43 & $4(5.5)$ & $9(12.3)$ & $1(1.3)$ & $8(10.9)$ & $4(5.5)$ & [49] \\
\hline Zhu L et al./China & $30.12 .19-20.03 .20$ & 7337 & 54 & $952(13)$ & $1763(24.0)$ & $363(5.0)$ & NR & $56(0.8)$ & [7] \\
\hline Guan W et al./China & $11.12 .20-31.01 .20$ & 1590 & 48.9 & $130(8.2)$ & $269(16.9)$ & $59(3.7)$ & $111(7)$ & $24(1.5)$ & {$[50]$} \\
\hline Wang L et al./China & $01.01 .20-06.02 .20$ & 339 & 69 & $54(16.0)$ & $138(40.8)$ & $53(15.7)$ & NR & $21(6.2)$ & {$[51]$} \\
\hline Zhou X et al./China & $25.01 .20-20.02 .20$ & 110 & 57.7 & $11(10.0)$ & $36(32.7)$ & $10(9.1)$ & NR & $3(2.7)$ & [52] \\
\hline Huang R et al./China & $22.01 .20-10.02 .20$ & 202 & 44 & $19(9.4)$ & $29(14.4)$ & $5(2.5)$ & $16(7.9)$ & $7(3.5)$ & [53] \\
\hline Guo W et al./China & $10.02 .20-29.02 .20$ & 174 & 59 & $37(21.26)$ & $43(24.7)$ & $32(18.4)$ & NR & $14(9.7)$ & {$[6]$} \\
\hline Orioli L et al./Belgium & $29.02 .20-26.04 .20$ & 11018 & NR & NR (21.2) & NR (38.5) & NR (32.9) & NR & NR (14.5) & [54] \\
\hline Du Y et al./China & 09.01.20-15.02.20 & 85 & 65.8 & $19(22.4)$ & $32(37.6)$ & $10(11.8)$ & NR & $2(2.4)$ & {$[55]$} \\
\hline Li T et al./China & 01.01.20-31.01.20 & 182 & 65.8 & $51(28.0)$ & $29(15.9)$ & $19(10.4)$ & NR & $15(8.24)$ & {$[56]$} \\
\hline Palaiodimos L et al./USA & 09.03.20-12.04.20 & 200 & 64 & $79(39.5)$ & $152(76.0)$ & $33(16.5)$ & $65(32.5)$ & $28(14.0)$ & [57] \\
\hline
\end{tabular}

Table 1: Prevalence of diabetes, hypertension, cardiovascular disease, smoking status, and respiratory diseases in COVID-19. *: Patients with severe or fatal infection and acute exacerbations have been included, \#: only tracheal intubated patients included, $\because$ hospitalized and non-hospitalized patients included, $\because$ only fatal cases have been reported, $\because:$ only old patients have been included, $€$ : evaluated according to reported number and ratios, ${ }^{\beta}$ : only COVID-19 patients are included, ${ }^{\alpha}:$ done with hemodialysis patients, CVD: cardiovascular disease, HT: hypertension, NR: not reported, RD: respiratory disease, Ref: references, USA: United States of America. 


\begin{tabular}{|l|l|l|l|l|l|l|}
\hline Author & $\mathbf{n}$ & $\begin{array}{l}\text { DM } \\
\mathbf{n}(\boldsymbol{\%})\end{array}$ & $\begin{array}{l}\text { Mild-moderate } \\
\text { cases n }(\boldsymbol{\%})\end{array}$ & $\begin{array}{l}\text { Severe cases } \\
\mathbf{n}(\boldsymbol{\%})\end{array}$ & $\begin{array}{l}\mathbf{p} \text { value between mild- } \\
\text { moderate and severe cases }\end{array}$ & Ref \\
\hline Ren H et al. & 151 & $39(25.8)$ & $16 / 89(18.0)$ & $23 / 62(37.1)$ & 0.008 & {$[13]$} \\
\hline Yuan J et al. & 94 & $5(5.30)$ & $3 / 75(4)$ & $2 / 11(18.18)$ & NR & {$[14]$} \\
\hline Chen Q et al. & 145 & $14(9.65)$ & $7 / 102(6.9)$ & $7 / 43(16.3)$ & 0.08 & {$[15]$} \\
\hline Huang Q et al. & 54 & $5(9.3)$ & $3 / 51(5.9)$ & $2 / 3(66.7)$ & NR & {$[18]$} \\
\hline Zhao XY et al. & 91 & $3(3.3)$ & $2 / 61(3.27)$ & $1 / 30(3.3)$ & NR & {$[19]$} \\
\hline Ren D et al. & 150 & $12(8)$ & $3 / 101(3)$ & $9 / 49(18.4)$ & 0.003 & {$[20]^{\mp}$} \\
\hline Shi Y et al. & 487 & $29(6.0)$ & $22 / 438(5.0)$ & $7 / 49(14.3)$ & 0.009 & {$[27]$} \\
\hline Wan S et al. & 135 & $12(8.9)$ & $3 / 95(3.1)$ & $9 / 40(22.5)$ & NR & {$[32]$} \\
\hline Huang C et al. & 41 & $8(20)$ & $7 / 28(25)$ & $1 / 13(8)$ & 0.16 & {$[34]$} \\
\hline Xiong F et al. & 131 & $30(22.9)$ & $25 / 101(24.8)$ & $5 / 30(16.7)$ & 0.35 & {$[35]^{\alpha}$} \\
\hline Wu C et al. & 201 & $22(10.9)$ & $6 / 117(5.1)$ & $16 / 84(19.0)$ & 0.002 & {$[36]$} \\
\hline Itelman E et al. & 162 & $30(18.5)$ & $22 / 136(16.17)$ & $8 / 26(30.8)$ & 0.04 & {$[37]^{\epsilon}$} \\
\hline Chow N et al. & 7162 & $784(10.9)$ & $251 / 1037(24)^{*}$ & $148 / 457(32)^{*}$ & NR & {$[38]$} \\
\hline Hong KS et al. & 98 & $9(9.2)$ & $6 / 85(7.1)$ & $3 / 13(23.1)$ & 0.096 & {$[41]$} \\
\hline Zhang J et al. & 140 & $17(12.1)$ & $9 / 82(11.0)$ & $8 / 58(13.8)$ & 0.615 & {$[46]$} \\
\hline Wang X et al. & 1012 & $27(2.7)$ & $20 / 912(2.2)$ & $7 / 100(7.0)$ & 0.01 & {$[47]$} \\
\hline Zheng Y et al. & 73 & $4(5.5)$ & $1 / 43(2.3)$ & $3 / 30(10)$ & NR & {$[49]$} \\
\hline Huang R et al. & 202 & $19(9.4)$ & $11 / 179(6.1)$ & $8 / 23(34.8)$ & $<0.001$ & {$[53]$} \\
\hline
\end{tabular}

Table 2: Prevalence of diabetes in non-severe and severe COVID-19 patients. ${ }^{*}$ : only hospitalized patients included, ${ }^{\ddagger}$ : sepsis and non-sepsis patients are evaluated, ${ }^{\alpha}$ : done with hemodialysis patients, ${ }^{\epsilon}$ : evaluated according to reported number and ratios, DM: diabetes mellitus, NR: not reported, Ref: references.

\begin{tabular}{|l|l|l|l|l|l|l|}
\hline Author & $\mathbf{n}$ & $\begin{array}{l}\text { DM } \\
\mathbf{n}(\boldsymbol{\%})\end{array}$ & $\begin{array}{l}\text { Survivor } \\
\mathbf{n}(\boldsymbol{\%})\end{array}$ & $\begin{array}{l}\text { Non-survivor } \\
\mathbf{n}(\boldsymbol{\%})\end{array}$ & p value & Ref \\
\hline Tu W et al. & 174 & $17(9.77)$ & $11 / 149(7.4)$ & $6 / 25(24.0)$ & 0.010 & {$[16]$} \\
\hline Wang D et al. & 107 & $11(10.3)$ & $6 / 88(6.8)$ & $5 / 19(26.3)$ & 0.024 & {$[17]$} \\
\hline Deng G et al. & 44,672 & $1102(5.3)$ & $80 / 406(19.7)$ & $1022 / 20576(5.0)$ & $<0.001$ & {$[21]^{*}$} \\
\hline Zhao X et al. & 532 & $59(11.09)$ & $50 / 503(9.9)$ & $9 / 29(31.0)$ & $<0.001$ & {$[23]^{\epsilon}$} \\
\hline Zhang J et al. & 19 & $4(21.05)$ & $1 / 11(9.09)$ & $3 / 8(37.5)$ & NR & {$[28]$} \\
\hline Ruan Q et al. & 150 & $25(16.7)$ & $13 / 82(15.85)$ & $12 / 68(17.6)$ & 0.88 & {$[29]$} \\
\hline Nikpouraghdam M et al. & 2964 & $113(3.81)$ & $102 / 2725(3.74)$ & $11 / 239(4.6)$ & NR & {$[33]^{\epsilon}$} \\
\hline Zhou F et al. & 191 & $36(19)$ & $19 / 137(14)$ & $17 / 54(31)$ & 0.0051 & {$[43]$} \\
\hline Wang L et al. & 339 & $54(16.0)$ & $43 / 274(15.8)$ & $11 / 65(17.2)$ & 0.116 & {$[51]^{:}$} \\
\hline
\end{tabular}

Table 3: Prevalence of diabetes in survivor and non-survivor COVID-19 patients. *: the fatality rate is detected after missing data is evaluated, $\because:$

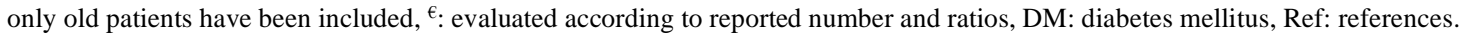

\section{References}

1. Xu G, Yang Y, Du Y, et al. Clinical pathway for early diagnosis of COVID-19: Updates from experience to evidence-based practice. Clin Rev Allergy Immunol. 2020;24(1)1-12.

2. Johns Hopkins University and Medicine Coronavirus Research Center. COVID-19 Dashboard by CSSE. 2020.

3. World Health Oganization. Diabetes. 2020.

4. Zimmet P, Alberti KG, Magliano DJ, et al. Diabetes mellitus statistics on prevalence and mortality: facts and fallacies. Nat Rev Endocrinol. 2016.12(10):616-22. 
5. Gürbüz P, Düzova H, Yildiz A, et al. Effects of Noopept on cognitive functions and pubertal process in rats with diabetes. Life Sci. 2019;233(1):116698.

6. Guo W, Li M, Dong Y, et al. Diabetes is a risk factor for the progression and prognosis of COVID-19. Diabetes Metab Res Rev. 2020;31(1):e3319.

7. Zhu 1, She Z, Cheng X, et al. Association of blood glucose control and outcomes in patients with COVID-19 and pre-existing type 2 diabetes. Cell Metab. 2020;31(6):1068-77.

8. Katulanda P, Dissanayake HA, Ranathunga I, et al. Prevention and management of COVID-19 among patients with diabetes: An appraisal of the literature. Diabetologia. 2020;63(8): 1440-452.

9. Torres A, Blasi T, Dartois N, et al. Which individuals are at increased risk of pneumococcal disease and why? Impact of COPD, asthma, smoking, diabetes, and/or chronic heart disease on community-acquired pneumonia and invasive pneumococcal disease. Thorax. 2015;70(10):984-89.

10. Muniyappa R, Gubbi S. COVID-19 pandemic, coronaviruses, and diabetes mellitus. Am J Physiol Endocrinol Metab. 2020;318(5):E736-E741.

11. Zheng Z, Peng F, Xu B, et al. Risk factors of critical \& mortal COVID-19 cases: A systematic literature review and meta-analysis. J Infect. 2020;4453(20):30234-236.

12. Lian J, Cai H, Hao S, et al. Comparison of epidemiological and clinical characteristics of COVID-19 patients with and without Wuhan exposure history in Zhejiang Province, China*. J Zhejiang Univ Sci B. 2020;21(5):369-377.

13. Ren H, Yang Y, Wang F, et al. Association of the insulin resistance marker TyG index with the severity and mortality of COVID-19. Cardiovasc Diabetol. 2020;19(1):58.

14. Yuan J, Zou R, Zeng L, et al. The correlation between viral clearance and biochemical outcomes of 94 COVID-19 infected discharged patients. Inflamm Res. 2020;69(1):599-606.

15. Chen Q, Zheng Z, Zhang C, et al. Clinical characteristics of 145 patients with corona virus disease 2019 (COVID-19) in Taizhou, Zhejiang, China. Infection. 2020;27(1):1-9.

16. Tu W, Cao J, Yu L, et al. Clinicolaboratory study of 25 fatal cases of COVID-19 in Wuhan. Intensive care med. 2020;46(6): 1117-120.

17. Wang D, Yin Y, Hu C, et al. Clinical course and outcome of 107 patients infected with the novel coronavirus, SARS-CoV-2, discharged from two hospitals in Wuhan, China. Crit Care. 2020;24(1):188.

18. Huang Q, Deng X, Li Y, et al. Clinical characteristics and drug therapies in patients with the common-type coronavirus disease 2019 in Hunan, China. Int J Clin Pharm. 2020;28(1):1-9.

19. Zhao X, Xu X, Yin H, et al. Clinical characteristics of patients with 2019 coronavirus disease in a non-Wuhan area of Hubei Province, China: a retrospective study. BMC Infect Dis. 2020;20(1):311.

20. Ren D, Ren C, Yao R, et al. Clinical features and development of sepsis in patients infected with SARSCoV-2: A retrospective analysis of 150 cases outside Wuhan, China. Intensive Care Med. 2020;15(1):1-4.

21. Deng G, Yin M, Chen X, et al. Clinical determinants for fatality of 44,672 patients with COVID-19. Crit Care. 2020;24(1):179.

22. Liu J, Chen T, Yang H, et al. Clinical and radiological changes of hospitalised patients with COVID-19 pneumonia from disease onset to acute exacerbation: a multicentre paired cohort study. Eur Radiol. 2020;8(1):1-7. 
23. Zhao X, Wang K, Zuo P, et al. Early decrease in blood platelet count is associated with poor prognosis in COVID-19 patients-indications for predictive, preventive, and personalized medical approach. EPMA J. 2020;11(2):1-7.

24. Helms J, Tacquard C, Severac F, et al. High risk of thrombosis in patients with severe SARS-CoV-2 infection: a multicenter prospective cohort study. Intensive Care Med. 2020;46(6):1089-98.

25. Pavoni V, Gianesello L, Pazzi M, et al. Evaluation of coagulation function by rotation thromboelastometry in critically ill patients with severe COVID-19 pneumonia. J Thromb Thrombolysis. 2020;11(1):1-6.

26. Zhang L, Li J, Zhou M, et al. Summary of 20 tracheal intubation by anesthesiologists for patients with severe COVID-19 pneumonia: retrospective case series. J Anesth. 2020;17(1):1-8.

27. Shi Y, Yu X, Zhao H, et al. Host susceptibility to severe COVID-19 and establishment of a host risk score: findings of 487 cases outside Wuhan. Crit Care. 2020;24(1):108.

28. Zhang J, Liu P, Wang M, et al. The clinical data from 19 critically ill patients with coronavirus disease 2019: a single-centered, retrospective, observational study. Z Gesundh Wiss. 2020;21(1)1-4.

29. Ruan Q, Yang K, Wang W, et al. Clinical predictors of mortality due to COVID-19 based on an analysis of data of 150 patients from Wuhan, China. Intensive Care Med. 2020;46(5):846-48.

30. Gentile S, Strollo F, Ceriello A. The need for diabetes care customization in the ICU at the time of SARSCoV-2 outbreak. Diabetes Ther. 2020;11(6):1-3.

31. Lian J, Jin X, Hao S, et al. Epidemiological, clinical, and virological characteristics of 465 hospitalized cases of coronavirus disease 2019 (COVID-19) from Zhejiang province in China. Influenza Other Respir Viruses. 2020;12(1):1-11.

32. Wan S, Xiang Y, Fang W, et al. Clinical features and treatment of COVID-19 patients in northeast Chongqing. J Med Virol. 2020;92(7):797-806.

33. Nikpouraghdam M, Farahani AJ, Alishiri GH, et al. Epidemiological characteristics of coronavirus disease 2019 (COVID-19) patients in IRAN: A single center study. J Clin Virol. 2020;127(1):104378.

34. Huang C, Wang Y, Li X. et al. Clinical features of patients infected with 2019 novel coronavirus in Wuhan, China. Lancet. 2020;395(10223):497-506.

35. Xiong F, Tang H, Liu L, et al. Clinical characteristics of and medical interventions for COVID-19 in hemodialysis patients in Wuhan, China. J Am Soc Nephrol. 2020;31(7):1387-397.

36. Wu C, Chen X, Cai Y, et al. Risk factors associated with acute respiratory distress syndrome and death in patients with coronavirus disease 2019 pneumonia in Wuhan, China. JAMA Intern Med. 2020;180(7):1-11.

37. Itelman E, Wasserstrum Y, Segev A, et al. Clinical characterization of 162 COVID-19 patients in Israel: Preliminary report from a large tertiary center. Isr Med Assoc J. 2020;22(5)271-74.

38. Preliminary estimates of the prevalence of selected underlying health conditions among patients with coronavirus disease 2019 - United States, February 12-March 28, 2020. MMWR Morb Mortal Wkly Rep 2020;69:382-386.

39. Leung C. Clinical features of deaths in the novel coronavirus epidemic in China. Rev Med Virol. 2020;16(1):e2103. 
40. de Abajo FJ, Rodríguez-Martín S, Lerma V, et al. Use of renin-angiotensin-aldosterone system inhibitors and risk of COVID-19 requiring admission to hospital: a case-population study. Lancet. 2020;395(10238):17051714.

41. Hong KS, Lee KH, Chung JH, et al. Clinical features and outcomes of 98 patients hospitalized with SARSCoV-2 Infection In Daegu, South Korea: A brief descriptive study. Yonsei Med J. 2020;61(5):431-37.

42. Richardson S, Hirsch JS, Narasimhan M, et al. Presenting characteristics, comorbidities, and outcomes among 5700 patients hospitalized with COVID-19 in the New York City area. Jama. 2020;323(20):2052-59.

43. Zhou F, Yu T, Du R, et al. Clinical course and risk factors for mortality of adult inpatients with COVID-19 in Wuhan, China: a retrospective cohort study. Lancet. 2020;395(10229):1054-62.

44. Li X, Wang L, Yan S, et al. Clinical characteristics of 25 death cases with COVID-19: a retrospective review of medical records in a single medical center, Wuhan, China. Int J Infect Dis. 2020;94(1):128-32.

45. Garg S, Kim L, Whitaker M, et al. Hospitalization rates and characteristics of patients hospitalized with laboratory-confirmed coronavirus disease 2019 - COVID-NET, 14 States, March 1-30, 2020. MMWR Morb Mortal Wkly Rep 2020;69(1):458-464.

46. Zhang J, Dong X, Cao Y, et al. Clinical characteristics of 140 patients infected with SARS-CoV-2 in Wuhan, China. Allergy. 2020;75(7):1730-741.

47. Wang X, Fang J, Zhu Y, et al. Clinical characteristics of non-critically ill patients with novel coronavirus infection (COVID-19) in a Fangcang Hospital. Clin Microbiol Infect. 2020;S1198-743X(20):1-6.

48. Bode B, Garrett V, Messler J, et al. Glycemic characteristics and clinical outcomes of COVID-19 patients hospitalized in the United States. J Diabetes Sci Technol. 2020;14(4):813-21.

49. Zheng Y, Xiong C, Liu Y, et al. Epidemiological and clinical characteristics analysis of COVID-19 in the surrounding areas of Wuhan, Hubei province in 2020. Pharmacol Res. 2020;157(1):104821.

50. Guan W-J, Liang W-H, Zhao Y, et al. Comorbidity and its impact on 1590 patients with Covid-19 in China: A nationwide analysis. Eur Respir J. 2020;55(5): 2000547.

51. Wang L, He W, Yu X, et al. Coronavirus disease 2019 in elderly patients: Characteristics and prognostic factors based on 4-week follow-up. J Infect. 2020;80(6):639-45.

52. Zhou X, Zhu J, Xu T. Clinical characteristics of coronavirus disease 2019 (COVID-19) patients with hypertension on renin-angiotensin system inhibitors. Clin Exp Hypertens. 2020;42(7):656-60.

53. Huang R, Zhu L, Xue L, et al. Clinical findings of patients with coronavirus disease 2019 in Jiangsu province, China: A retrospective, multi-center study. PLoS Negl Trop Dis. 2020;14(5): e0008280.

54. Orioli L, Hermans MP, Thissen J-P, et al. COVID-19 in diabetic patients: related risks and specifics of management. Ann Endocrinol (Paris). 2020;81(2-3)101-109.

55. Du, Y., et al., Clinical features of 85 fatal cases of COVID-19 from Wuhan. A retrospective observational study. Am J Respir Crit Care Med. 2020;201(11):1372-379.

56. Li T, Zhang Y, Gong C, et al. Prevalence of malnutrition and analysis of related factors in elderly patients with COVID-19 in Wuhan, China. Eur J Clin Nutr. 2020;74(6):871-75. 
57. Palaiodimos L, Kokkinidis DG, Li W, et al. Severe obesity, increasing age and male sex are independently associated with worse in-hospital outcomes, and higher in-hospital mortality, in a cohort of patients with COVID-19 in the Bronx, New York. Metabolism. 2020;108(1):154262.

58. Martins IJ. COVID-19 infection and anti-aging gene inactivation. Acta Scientific Nutritional Health. 2020;4(5):1-2.

59. Xiao E, Mattos M, Vieira GHA, et al. Diabetes enhances IL-17 expression and alters the oral microbiome to increase its pathogenicity. Cell Host Microbe. 2017;22(1): p. 120-128.

60. Kim EJ, Ha KH, Kim DJ, et al. Diabetes and the risk of infection: A national cohort study. Diabetes Metab J. 2019;43(6):804-14.

61. Ahuja W, Kumar N, Kumar S, et al. Precipitating risk factors, clinical presentation, and outcome of diabetic ketoacidosis in patients with type 1 diabetes. Cureus. 2019;11(5):e4789.

62. Chan KH, Thimmareddygari D, Ramahi A, et al. Clinical characteristics and outcome in patients with combined diabetic ketoacidosis and hyperosmolar hyperglycemic state associated with covid-19: a retrospective, hospital-based observational case series. Diabetes Res Clin Pract. 2020;166(1):108279.

63. Kim N-Y, Ha E, Moon JS, et al. Acute hyperglycemic crises with Coronavirus Disease-19: Case Reports. Diabetes Metab J. 2020;44(2):349-53.

64. Li J, Wang X, Chen J, et al. COVID-19 infection may cause ketosis and ketoacidosis. Diabetes Obes Metab. 2020; ePub:ahead of print.

65. Nunes JP. Arterial hypertension and sepsis. Portuguese Journal of Cardiology. 2003;22(11):1375-379.

66. Badawi A, Ryoo SG. Prevalence of comorbidities in the Middle East respiratory syndrome coronavirus (MERS-CoV): A systematic review and meta-analysis. Int J Infect Dis. 2016;49(1):129-33.

67. Garbati MA, Fagbo SF, Fang VJ, et al. A comparative study of clinical presentation and risk factors for adverse outcome in patients hospitalised with acute respiratory disease due to MERS coronavirus or other causes. PLoS One. 2016;11(11):e0165978.

68. Stang A, Standl F, Jöckel K-H. Characteristics of COVID-19 pandemic and public health consequences. Herz. 2020;45(4):313-15.

69. Yu X, Sun S, Shi Y, et al. SARS-CoV-2 viral load in sputum correlates with risk of COVID-19 progression. Crit Care. 2020;24(1):170.

70. Moccia F, Gerbino A, Lionetti V, et al. COVID-19-associated cardiovascular morbidity in older adults: A position paper from the Italian society of cardiovascular researches. Geroscience. 2020;20(1):1-29.

71. Shi Y, Wang G, Cai X-P, et al. An overview of COVID-19. J Zhejiang Univ Sci. 2020;21(5):343-60. 